\title{
Edge-colorings of 4-regular graphs with the minimum number of palettes
}

\author{
S.Bonvicini* G.Mazzuoccolo $^{\dagger}$
}

July 9, 2014

\begin{abstract}
A proper edge-coloring of a graph $G$ is an assignment of colors to the edges of $G$ such that adjacent edges receive distinct colors. A proper edge-coloring defines at each vertex the set of colors of its incident edges. Following the terminology introduced by Horňák, Kalinowski, Meszka and Woźniak, we call such a set of colors the palette of the vertex. What is the minimum number of distinct palettes taken over all proper edge-colorings of G? A complete answer is known for complete graphs and cubic graphs. We study in some detail the problem for 4-regular graphs.
\end{abstract}

Keywords: palette index, 4-regular graphs, edge-coloring, even cycle decomposition, even 2-factor. $M S C(2010)$ : $05 C 15$

\section{Introduction}

Throughout this paper, a graph $G$ always means a simple finite graph (without loops and parallel edges). We refer to any introductory book for graphtheoretical notation and terminology not described in this paper (see for instance $[1])$.

An edge-coloring of a graph $G$ is an assignment of colors to the edges of $G$ : it is proper if adjacent edges receive distinct colors. The minimum number of colors used in a proper edge-coloring of $G$ is the chromatic index of $G$ and is denoted by $\chi^{\prime}(G)$. By Vizing's Theorem, $\Delta(G) \leq \chi^{\prime}(G) \leq \Delta(G)+1$, where $\Delta(G)$ is the maximum degree of $G$. A graph is said to be class 1 if $\chi^{\prime}(G)=\Delta(G)$ and class 2 if $\chi^{\prime}(G)=\Delta(G)+1$.

Let $f$ be a proper edge-coloring of $G$ and let $v$ be a vertex of $G$. Denote by $P_{f}(v)$ the set of colors assigned by $f$ to the edges incident to $v$. The set $P_{f}(v)$ is called the palette of $v$ (with respect to $f$ ). For every proper edge-coloring of

\footnotetext{
*Dipartimento di Scienze e Metodi dell'Ingegneria, Università di Modena e Reggio Emilia, via Amendola 2, 42100 Reggio Emilia (Italy)

${ }^{\dagger}$ Dipartimento di Scienze Fisiche, Informatiche e Matematiche, Università di Modena e Reggio Emilia, via Campi 213/b, 41126 Modena (Italy)
} 
$G$ it is possible to define the set $\mathcal{P}_{f}=\cup_{v \in V(G)} P_{f}(v)$. The set $\mathcal{P}_{f}$ is the set of distinct palettes of $f$ and its cardinality is at most $|V(G)|$.

Definitions similar to that of $\mathcal{P}_{f}(v)$ can be given also for vertex-colorings (see [5] and [8]). These definitions offer a wide range of problems. For instance, some authors study the problem of finding a vertex-distinguish proper edge-coloring with the minimum number of colors (see [4] or [6]). A proper edge-coloring of a graph $G$ is said to be vertex-distinguish if distinct vertices of $G$ have distinct palettes. The set $\mathcal{P}_{f}$ of a vertex-distinguish coloring has cardinality $|V(G)|$, that is, $\mathcal{P}_{f}$ is as big as possible. Here, we are looking for proper edge-colorings with $\mathcal{P}_{f}$ as small as possible. As far as we know, this kind of proper colorings have been studied for the first time in [7], where the authors define the palette index, denoted by $\check{s}(G)$, of a simple graph $G$ as follows:

$$
\check{s}(G)=\min \left\{\left|\mathcal{P}_{f}\right|: f \text { proper edge-coloring of } G\right\}
$$

In [7] the authors also determine the palette index of cubic graphs and complete graphs and observe that the palette index of a $d$-regular graph is 1 if and only if the graph is class 1 . A classical result by Robinson and Wormald [11] assures that, for any fixed $d \geq 3$, almost all $d$-regular graphs of even order are class 1 . This result is equivalent to say that almost all $d$-regular graphs of even order have palette index 1.

What about the palette index of class $2 d$-regular graphs?

It is shown in [7] that the palette index of a regular graph is different from 2. Furthermore, the palette index of a $d$-regular graph is at most $d+1$ : by Vizing's Theorem, a $d$-regular graph $G$ of class 2 possesses a proper edge-coloring $f$ whose color-set $\mathcal{C}$ has cardinality $d+1$; since each palette is a $d$-subset of $\mathcal{C}$, the cardinality of $\mathcal{P}_{f}$ is at most $\left(\begin{array}{c}d+1 \\ d\end{array}\right)$, that is, $\check{s}(G) \leq d+1$. Therefore, the palette index of a class $2 d$-regular graph satisfies the inequalities

$$
3 \leq \check{s}(G) \leq d+1 .
$$

We wonder whether this upper bound for the palette index of a $d$-regular graph is really achieved. In other words, we wonder whether, for any fixed $d \geq 2$, a $d$-regular graph with palette index $d+1$ does exist.

For $d=2$ and 3 there exist $d$-regular graphs with palette index 3 and 4 , respectively. We can consider a cycle with at least one circuit of odd length for $d=2$, and a cubic graph with no perfect matching for $d=3$ (see [7]).

In the present paper, we consider the case $d=4$. We will make use of the following standard definitions: a circuit is a 2-regular connected graph and a cycle is a 2-regular graph whose connected components are circuits. A spanning cycle of a graph $G$ is a 2-factor of $G$. A cycle (a 2-factor) is even if it has no circuit of odd length. An even cycle decomposition of a graph $G$ is a partition $\mathcal{E}$ of the edge-set of $G$ into even cycles. If $\mathcal{E}$ consists of $m$ cycles, then we say that $G$ has an even cycle decomposition of size $m$.

It is conjectured in [10] that a random 4-regular graph of order $2 n+1$ asymptotically almost surely decomposes into a circuit of length $2 n$ and two other circuits of even length intersecting in exactly one vertex. In other words, 
a random 4-regular graph of odd order asymptotically almost surely has an even cycle decomposition of size 3. From an even cycle decomposition of size 3 of a 4-regular graph $G$ we can easily obtain a proper edge-coloring of $G$ with exactly 3 palettes (see Proposition 11). Hence, almost all 4-regular graphs should have palette index 1 or 3 . Hence, 4-regular graphs with palette index 4 and 5, in particular the connected ones, should be rare.

In Section 2.2, we construct non-connected 4-regular graphs with palette index 4 and 5 . The construction of connected 4-regular graphs with palette index 5 turns out to be harder to achieve than non-connected case. Nevertheless, we are able to furnish in Proposition 9 a construction of an infinite family of 4-regular graphs with palette index 5 .

The concept of $\check{s}$-minimal coloring (see Section 2.1 for a definition) is relevant in the study of 4-regular graphs with large palette index. A detailed study of $\check{s}$-minimal colorings allows us to say that in a 4-regular graph $G$ with palette index 3 at least one of the following cases occurs:

(i) $G$ has an even cycle decomposition of size 3 ;

(ii) $G$ has an even 2-factor.

By this result, a 4-regular graph with palette index 3 and no perfect matching always admits an even cycle decomposition of size 3. We can extend this property to $4 r$-regular graphs: we can prove that a $4 r$-regular graph, $r \geq 1$, with palette index 3 and no perfect matching has an even cycle decomposition of size $3 r$ (see Section 3).

In Section 3 we also note that the family of 4-regular graphs with an even cycle decomposition of size $m \leq 3$ is strictly contained in the family of 4-regular graphs with palette index $\leq 3$. We do not know whether all 4-regular graphs with an even cycle decomposition have palette index $\leq 3$ or not, but we can prove that a 4-regular graph with an even cycle decomposition and palette index $>3$ has no even cycle decomposition of size $m \leq 3$ (see the remarks in Section 3). A natural question about even cycle decompositions of 4-regular graphs arise from these considerations:

Does there exist a 4-regular graph with all even cycle decompositions of size larger than 3 ?

In Section 2.3 we also study connected 4-regular graphs with palette index 4. A 4-regular graph with palette index 4 might or might not have a perfect matching. We can construct many examples of 4-regular graphs with no perfect matching and palette index 4 (see Proposition 6). The construction of a 4regular graph with a perfect matching and palette index 4 is more complicated: we exhibit an example in Proposition 8. Finally, we note that a 4-regular graph with a perfect matching and palette index 4 has no even 2-factor, nevertheless the non-existence of an even 2-factor does not guarantee that the graph has palette index $>3$, see Proposition 7 .

In the last section we list some open problems. In particular, we leave as open problems the construction of a $d$-regular graph with palette index $d+1$ for every integer $d \geq 5$. 


\section{4-regular graphs.}

As already remarked in the introduction, the palette index of a class $2 d$-regular graph $G$ satisfies the inequalities $3 \leq \check{s}(G) \leq d+1$. We recall that $\check{s}(G)=1$ if and only if $G$ is of class 1 and a simple counting argument excludes the case $\check{s}(G)=2$ (see [7]). For a 4-regular graph of class 2 the previous inequalities become $3 \leq \check{s}(G) \leq 5$. In this section we construct 4-regular graphs with palette index 5 . First of all, we show that the disjoint union of two graphs could have palette index larger than the maximum between the palettes indices of the two graphs. In particular, we show that the palette index of a non-connected 4-regular graph, with at least two connected components having palette index 3 , might be bigger than 3 . We use this fact to construct non-connected 4-regular graphs with palette index 4 and 5 (see Section 2.2). Finally, in Section 2.3 we consider the connected case.

\section{$2.1 \check{s}$-minimal colorings}

Given a graph $G$, we denote by $\mathcal{F}(G)$ the set of proper edge-colorings of $G$ having the minimum number of palettes $\check{s}(G)$. The elements of $\mathcal{F}(G)$ will be called $\check{s}$-colorings. We say that an $\check{s}$-coloring $f \in \mathcal{F}(G), f: \mathcal{C} \rightarrow E(G)$, is $\check{s}$ minimal if $|\mathcal{C}| \leq\left|\mathcal{C}^{\prime}\right|$ for every $f^{\prime} \in \mathcal{F}(G)$, with $f^{\prime}: \mathcal{C}^{\prime} \rightarrow E(G)$. We recall that an edge-coloring using $\chi^{\prime}(G)$ colors is called minimum. As remarked in [7], a minimum edge-coloring might not be $\check{s}$-minimal.

We say that two edge-colorings $f: \mathcal{C} \rightarrow E(G)$ and $f^{\prime}: \mathcal{C}^{\prime} \rightarrow E(G)$ with set of palettes $\mathcal{P}_{f}$ and $\mathcal{P}_{f^{\prime}}$, respectively, are equivalent if there exists a bijection $\alpha: \mathcal{C} \rightarrow \mathcal{C}^{\prime}$ such that $\alpha(P) \in \mathcal{P}_{f^{\prime}}$ for every $P \in \mathcal{P}_{f}$. If we need to specify the permutation $\alpha$, then we will say that $f$ and $f^{\prime}$ are equivalent by the bijection $\alpha$.

Lemma 1. Let $G$ be a d-regular graph with palette index $\check{s}(G)$ and let $f$ be an $\check{s}$-minimal coloring of $G$ with color-set $\mathcal{C}$. Let $n(a)$ be the number of palettes of $f$ containing the color $a \in \mathcal{C}$. The followings hold:

(i) $\lceil(|\mathcal{C}|-1) /(d-1)\rceil \leq n(a) \leq \check{s}(G)$;

(ii) $\sum_{a \in \mathcal{C}} n(a)=d \cdot \check{s}(G)$;

(iii) $(|\mathcal{C}|-1)|\mathcal{C}| \leq d(d-1) \check{s}(G)$.

Proof. We denote by $\mathcal{R}$ the multiset defined by $\mathcal{R}=\cup_{P \in \mathcal{P}_{f}}\{\{a, b\}:\{a, b\} \subset$ $P\}$ and by $\mathcal{C}^{2}$ the set of all possible 2 -subsets of $\mathcal{C}$. We show that $\mathcal{C}^{2} \subseteq \mathcal{R}$, that is, every 2-subset $\{a, b\} \in \mathcal{C}^{2}$ is a subset of at least one palette of $f$. Assume, on the contrary, that no palette of $f$ contains the 2 -subset $\{a, b\}$, then we can replace $a$ and $b$ by a unique color $c \notin \mathcal{C}$. We obtain a new proper edge-coloring $f^{\prime}$ with color-set $\mathcal{C}^{\prime}=(\mathcal{C} \backslash\{a, b\}) \cup\{c\}$ of cardinality $\left|\mathcal{C}^{\prime}\right|=|\mathcal{C}|-1$. The replacement of the colors $a$ and $b$ by the new color $c$ does not increase the number of palettes of $f$, that is, $\left|\mathcal{P}_{f^{\prime}}\right| \leq\left|\mathcal{P}_{f}\right|$. Since no proper edge-coloring of $G$ has less than $\check{s}(G)$ palettes and $\left|\mathcal{P}_{f}\right|=\check{s}(G)$, also the set $\mathcal{P}_{f^{\prime}}$ has cardinality $\check{s}(G)$, that is, 
$f^{\prime} \in \mathcal{F}(G)$. That yields a contradiction, since $\left|\mathcal{C}^{\prime}\right|<|\mathcal{C}|$ and $f$ is $\check{s}$-minimal. It is thus proved that $\mathcal{C}^{2} \subseteq \mathcal{R}$. The set inclusion relation $\mathcal{C}^{2} \subseteq \mathcal{R}$ means that, for every color $a \in \mathcal{C}$, the multiset $\mathcal{R}$ contains all the 2 -subsets $\{a, x\}$, with $x \in \mathcal{C} \backslash\{a\}$. Since each palette $P \in \mathcal{P}_{f}$ containing the color $a$ provides exactly $(d-1) 2$-subsets $\{a, x\}$, each color $a \in \mathcal{C}$ belongs to at least $\lceil(|\mathcal{C}|-1) /(d-1)\rceil$ palettes of $f$, that is, $n(a) \geq\lceil(|\mathcal{C}|-1) /(d-1)\rceil$. Obviously, $n(a) \leq \check{s}(G)$. It is straightforward to see that the relation $\sum_{a \in \mathcal{C}} n(a)=d \cdot \check{s}(G)$ holds, since each palette of $f$ contains exactly $d$ distinct colors and $\left|\mathcal{P}_{f}\right|=\check{s}(G)$.

We show that the inequality $(|\mathcal{C}|-1)|\mathcal{C}| \leq d(d-1) \check{s}(G)$ holds. Since $\mathcal{C}^{2} \subseteq \mathcal{R}$, the inequality $\left(\begin{array}{c}|\mathcal{C}| \\ 2\end{array}\right) \leq|\mathcal{R}|$ holds. Since each palette $P \in \mathcal{P}_{f}$ contains exactly $\left(\begin{array}{l}d \\ 2\end{array}\right)$ elements of $\mathcal{C}^{2}$ and $\left|\mathcal{P}_{f}\right|=\check{s}(G)$, the multiset $\mathcal{R}$ consists of exactly $\left(\begin{array}{l}d \\ 2\end{array}\right) \check{s}(G)$ elements of $\mathcal{C}^{2}$. Therefore, $\left(\begin{array}{c}|\mathcal{C}| \\ 2\end{array}\right) \leq|\mathcal{R}|=\left(\begin{array}{l}d \\ 2\end{array}\right) \check{s}(G)$, that is, $(|\mathcal{C}|-1)|\mathcal{C}| \leq d(d-$ $1) \check{s}(G)$.

As a consequence of Lemma 1, the following statements hold.

Proposition 1. Let $G$ be a 4-regular graph with palette index 3. Then every $\check{s}$-minimal coloring $f \in \mathcal{F}(G)$ has color-set of cardinality at most 6 .

Every š-minimal coloring of $G$ with 5 colors is equivalent to the edge-coloring $f_{1}$ with color-set $\mathcal{C}_{1}=\left\{a_{j}: 1 \leq j \leq 5\right\}$ and palettes $P_{1}=\left\{a_{1}, a_{2}, a_{3}, a_{4}\right\}$, $P_{2}=\left\{a_{1}, a_{2}, a_{3}, a_{5}\right\}, P_{3}=\left\{a_{1}, a_{2}, a_{4}, a_{5}\right\}$.

Every š-minimal coloring of $G$ whit 6 colors is equivalent to the edge-coloring $f_{2}$ with color-set $\mathcal{C}_{2}=\left\{a_{j}: 1 \leq j \leq 6\right\}$ and palettes $P_{1}=\left\{a_{1}, a_{2}, a_{3}, a_{4}\right\}$, $P_{2}=\left\{a_{1}, a_{2}, a_{5}, a_{6}\right\}, P_{3}=\left\{a_{3}, a_{4}, a_{5}, a_{6}\right\}$.

Proof. Let $f$ be an $\check{s}$-minimal coloring of $G$ with color-set $\mathcal{C}$ and $\mathcal{P}_{f}=\left\{P_{i}^{\prime}\right.$ : $1 \leq i \leq 3\}$. Since $G$ is class 2 , the cardinality of $\mathcal{C}$ is at least 5 ; by Lemma 1 , the cardinality of $\mathcal{C}$ satisfies the inequality $|\mathcal{C}|(|\mathcal{C}|-1) \leq 4 \cdot 3 \cdot 3$, that is, $5 \leq|\mathcal{C}| \leq 6$. We show that $f$ is equivalent to $f_{1}$ or $f_{2}$, according to whether the cardinality of $\mathcal{C}$ is 5 or 6 , respectively.

Consider $\mathcal{C}=\left\{b_{j}: 1 \leq j \leq 5\right\}$. We show that $f$ is equivalent to the edgecoloring $f_{1}$. By Lemma 1 , the relation $\sum_{b_{j} \in \mathcal{C}} n\left(b_{j}\right)=3 \cdot 4=12$, with $2 \leq n\left(b_{j}\right) \leq$ 3 , holds. From this relation one can see that exactly two colors of $\mathcal{C}$ belong to the three palettes of $f$, whereas the remaining three colors of $\mathcal{C}$ belong to exactly two palettes of $f$. Without loss of generality, we can set $n\left(b_{1}\right)=n\left(b_{2}\right)=3$ and $n\left(b_{j}\right)=2$ for $3 \leq j \leq 5$. Since the 2 -subset $\left\{b_{1}, b_{2}\right\}$ is contained in each $P_{i}^{\prime} \in \mathcal{P}_{f}$, each palette $P_{i}^{\prime}$ contains exactly one of the three 2 -subsets $\left\{b_{3}, b_{4}\right\}$, $\left\{b_{3}, b_{5}\right\},\left\{b_{4}, b_{5}\right\}$. Therefore, $\mathcal{P}_{f}=\left\{\left\{b_{1}, b_{2}, b_{3}, b_{4}\right\},\left\{b_{1}, b_{2}, b_{3}, b_{5}\right\},\left\{b_{1}, b_{2}, b_{4}, b_{5}\right\}\right\}$. It is straightforward to see that the colorings $f$ and $f_{1}$ are equivalent.

Consider $\mathcal{C}=\left\{b_{j}: 1 \leq j \leq 6\right\}$. We show that $f$ is equivalent to the edgecoloring $f_{2}$. By Lemma 1 , the relation $\sum_{b_{j} \in \mathcal{C}} n\left(b_{j}\right)=3 \cdot 4=12$, with $2 \leq n\left(b_{j}\right) \leq$ 3 , holds. From this relation one can see that $n\left(b_{j}\right)=2$ for every $b_{j} \in \mathcal{C}$. It is straightforward to see that at least two palettes of $f$ share two colors. Without loss of generality we can set $\left\{b_{1}, b_{2}\right\} \subset P_{1}^{\prime}, P_{2}^{\prime}$, whence $P_{3}^{\prime}=\left\{b_{3}, b_{4}, b_{5}, b_{6}\right\}$ and $\left\{P_{1}^{\prime}, P_{2}^{\prime}\right\}=\left\{\left\{b_{1}, b_{2}, a, b\right\},\left\{b_{1}, b_{2}, c, d\right\}\right\}$, with $\{a, b, c, d\}=\left\{b_{3}, b_{4}, b_{5}, b_{6}\right\}$. One can verify that the colorings $f$ and $f_{2}$ are equivalent by the bijection $\alpha: \mathcal{C} \rightarrow \mathcal{C}_{2}$ 
such that $\left\{\alpha\left(b_{1}\right), \alpha\left(b_{2}\right)\right\}=\left\{a_{1}, a_{2}\right\}$ and $\{\{\alpha(a), \alpha(b)\},\{\alpha(c), \alpha(d)\}\}=\left\{\left\{a_{3}, a_{4}\right\}\right.$, $\left.\left\{a_{5}, a_{6}\right\}\right\}$.

Corollary 1. Let $G$ be a 4-regular graph with palette index 3 . Then at least one of the following cases occurs:

(i) if $G$ has an edge-coloring $f_{1}$ with color-set $\mathcal{C}_{1}=\left\{a_{j}: 1 \leq j \leq 5\right\}$ and palettes $P_{1}=\left\{a_{1}, a_{2}, a_{3}, a_{4}\right\}, P_{2}=\left\{a_{1}, a_{2}, a_{3}, a_{5}\right\}, P_{3}=\left\{a_{1}, a_{2}, a_{4}, a_{5}\right\}$, then $G$ has an even 2 -factor;

(ii) if $G$ has an edge-coloring $f_{2}$ with color-set $\mathcal{C}_{2}=\left\{a_{j}: 1 \leq j \leq 6\right\}$ and palettes $P_{1}=\left\{a_{1}, a_{2}, a_{3}, a_{4}\right\}, P_{2}=\left\{a_{1}, a_{2}, a_{5}, a_{6}\right\}, P_{3}=\left\{a_{3}, a_{4}, a_{5}, a_{6}\right\}$, then $G$ has an even cycle decomposition of size 3 .

Proof. By Proposition 1, the graph $G$ has an $\check{s}$-minimal coloring $f \in \mathcal{F}(G)$ which is equivalent to the edge-coloring $f_{1}$ or $f_{2}$. If $f$ is equivalent to $f_{1}$, then the edges with colors $a_{1}$ and $a_{2}$ induce an even 2-factor of $G$. If $f$ is equivalent to $f_{2}$, then the edges with colors $a_{j}$ and $a_{j+1}$, with $j=1,3,5$, form a cycle $F_{j}$ with no circuit of odd length. The set $\left\{F_{1}, F_{3}, F_{5}\right\}$ is an even cycle decomposition of $G$ of size 3 .

Note that if $G$ admits both colorings $f_{1}$ and $f_{2}$, then $G$ has an even 2-factor and an even cycle decomposition of size 3 (see for instance the graph in Figure $1)$.

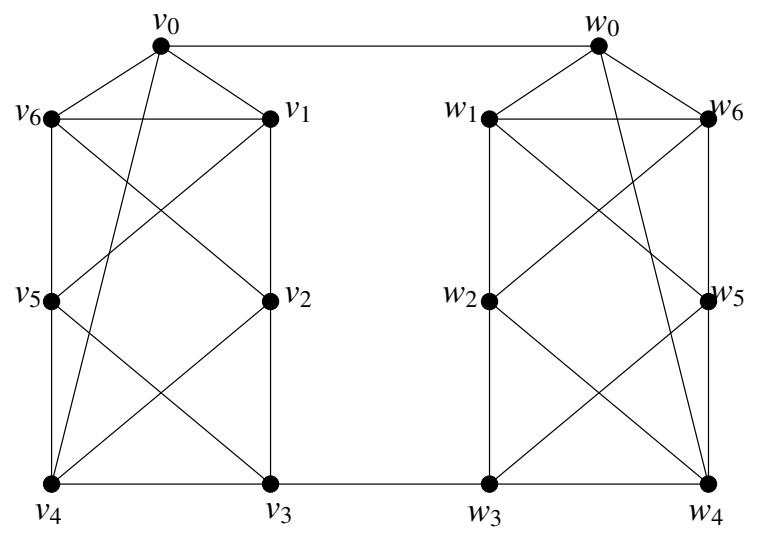

Figure 1: A 4-regular graph $G$ of class 2 with palette index 3 admitting both colorings $f_{1}$ and $f_{2}$; the graph $G$ has an even 2-factor consisting of the circuits $\left(v_{1}, v_{2}, v_{6}, v_{5}, v_{1}\right)$ and $\left(v_{0}, v_{4}, v_{3}, w_{3}, w_{4}, w_{5}, w_{6}, w_{2}, w_{1}, w_{0}, v_{0}\right)$; the graph $G$ also has an even cycle decomposition given by the cycles $F_{1}=\left\{\left(v_{0}, v_{4}, v_{5}, v_{6}, v_{0}\right)\right.$, $\left.\left(w_{0}, w_{4}, w_{5}, w_{6}, w_{0}\right)\right\}, \quad F_{2}=\left\{\left(v_{1}, v_{5}, v_{3}, v_{2}, v_{1}\right), \quad\left(w_{1}, w_{6}, w_{2}, w_{4}, w_{3}, w_{5}, w_{1}\right)\right\}$, $F_{3}=\left\{\left(v_{0}, w_{0}, w_{1}, w_{2}, w_{3}, v_{3}, v_{4}, v_{2}, v_{6}, v_{1}, v_{0}\right)\right\}$. 
Proposition 2. Let $G$ be a 4-regular graph with no perfect matching and $\check{s}(G)=$ 4. Then every $\check{s}$-minimal coloring of $G$ is equivalent to the edge-coloring $f_{3}$ having color-set $\mathcal{C}_{3}=\left\{a_{j}: 1 \leq j \leq 6\right\}$ and palettes $P_{1}=\left\{a_{1}, a_{2}, a_{3}, a_{4}\right\}$, $P_{2}=\left\{a_{1}, a_{2}, a_{3}, a_{5}\right\}, P_{3}=\left\{a_{1}, a_{2}, a_{4}, a_{6}\right\}, P_{4}=\left\{a_{3}, a_{4}, a_{5}, a_{6}\right\}$.

Proof. Let $f$ be an $\check{s}$-minimal coloring of $G$ with color-set $\mathcal{C}$ and $\mathcal{P}_{f}=\left\{P_{i}^{\prime}\right.$ : $1 \leq i \leq 4\}$. We denote by $\mathcal{R}$ the multiset $\mathcal{R}=\cup_{P \in \mathcal{P}_{f}}\{\{a, b\}:\{a, b\} \subset P\}$ and by $\mathcal{C}^{2}$ the set of all possible 2 -subsets of $\mathcal{C}$.

Since $G$ is a class 2 , the cardinality of $\mathcal{C}$ is at least 5 ; by Lemma $1,(|\mathcal{C}|-$ 1) $|\mathcal{C}| \leq 4 \cdot 3 \cdot 4$, that is, $5 \leq|\mathcal{C}| \leq 7$. By Lemma 1 , also the relation $\sum_{a \in \mathcal{C}} n(a)=$ $4 \cdot 4$, with $2 \leq n(a) \leq 4$, holds. Since $G$ has no perfect matching, each color $a \in \mathcal{C}$ belongs to at most three palettes of $f$, that is, $n(a) \leq 3$ for every $a \in \mathcal{C}$. From the relation $\sum_{a \in \mathcal{C}} n(a)=16$, with $2 \leq n(a) \leq 3$, one can see that $|\mathcal{C}| \geq 16 / 3$, that is, $6 \leq|\mathcal{C}| \leq 7$. We prove that $|\mathcal{C}|=6$. Suppose, on the contrary, that $|\mathcal{C}|=7$. We set $\mathcal{C}=\left\{b_{j}: 1 \leq j \leq 7\right\}$. From the relation $\sum_{b_{j} \in \mathcal{C}} n\left(b_{j}\right)=16$, with $2 \leq n(a) \leq 3$, one can see that exactly two colors of $\mathcal{C}$ belong to exactly three palettes of $f$, whereas the remaining five colors of $\mathcal{C}$ belong to exactly two palettes of $f$. Without loss of generality, we can set $n\left(b_{1}\right)=n\left(b_{2}\right)=3$ and $b_{1} \notin P_{4}^{\prime}$. Since $\left|P_{4}^{\prime}\right|=4$, at least one of the five colors in $\mathcal{C} \backslash\left\{b_{1}, b_{2}\right\}$ does not belong to $P_{4}^{\prime}$, say $b_{3}$. Therefore, the color $b_{3}$ belongs to exactly two of the palettes $P_{1}^{\prime}, P_{2}^{\prime}, P_{3}^{\prime}$, as $n\left(b_{3}\right)=2$. Since $b_{1}$ belongs to the three palettes $P_{1}^{\prime}$, $P_{2}^{\prime}, P_{3}^{\prime}$, the 2-subset $\left\{b_{1}, b_{3}\right\}$ is contained in exactly two of the palettes $P_{1}^{\prime}, P_{2}^{\prime}$, $P_{3}^{\prime}$. Consequently, the 2 -subset $\left\{b_{1}, b_{3}\right\}$ appears exactly twice in the multiset $\mathcal{R}$, that is, the multiset $\mathcal{R}$ does not contain the subset $\left\{\left\{b_{3}, x\right\}: x \in \mathcal{C}, x \neq b_{3}\right\}$ of $\mathcal{C}^{2}$. That yields a contradiction, since $\mathcal{C}^{2} \subseteq \mathcal{R}$ (see the proof of Lemma 1). It is thus proved that $|\mathcal{C}| \neq 7$, that is, $|\mathcal{C}|=6$.

Now, we show that $f$ is equivalent to $f_{3}$. We can always consider $\mathcal{C}_{3}=\mathcal{C}$, since $\left|\mathcal{C}_{3}\right|=|\mathcal{C}|$. From the relation $\sum_{a \in \mathcal{C}} n(a)=16$, with $2 \leq n(a) \leq 3$, one can see that four colors of $\mathcal{C}$ belong to exactly three palettes of $f$, whereas the remaining two colors of $\mathcal{C}$ belong to exactly two palettes of $f$. Without loss of generality, we can set $n\left(a_{j}\right)=3$ for $1 \leq j \leq 4$. First of all, we show that at least one of the possible 2-subsets of the set of colors $\left\{a_{1}, a_{2}, a_{3}, a_{4}\right\}$ is contained in three palettes of $f$. Suppose that this is not the case, then $\mathcal{P}_{f}=\left\{\left\{a_{1}, a_{2}, a_{3}, x_{1}\right\}\right.$, $\left.\left\{a_{1}, a_{2}, a_{4}, x_{2}\right\},\left\{a_{1}, a_{3}, a_{4}, x_{3}\right\},\left\{a_{2}, a_{3}, a_{4}, x_{4}\right\}\right\}$, with $\left\{x_{i}: 1 \leq i \leq 4\right\}=$ $\left\{a_{5}, a_{6}\right\}$. The 2 -subset $\left\{a_{5}, a_{6}\right\}$ does not belong to the multiset $\mathcal{R}$. That yields a contradiction, since $\mathcal{C}^{2} \subseteq \mathcal{R}$ (see the proof of Lemma 1 ). It is thus proved that at least one of the possible 2 -subsets of the set $\left\{a_{1}, a_{2}, a_{3}, a_{4}\right\}$ is contained in three palettes of $f$. Without loss of generality we can set $\left\{a_{1}, a_{2}\right\} \subset P_{1}^{\prime} \cap P_{2}^{\prime} \cap P_{3}^{\prime}$, whence $P_{4}^{\prime}=\left\{a_{3}, a_{4}, a_{5}, a_{6}\right\}$. Since $n\left(a_{3}\right)=n\left(a_{4}\right)=3$, we can set $P_{1}^{\prime}=\left\{a_{1}, a_{2}, a_{3}, a_{4}\right\}, P_{2}^{\prime}=\left\{a_{1}, a_{2}, a_{3}, x_{1}\right\}$ and $P_{3}^{\prime}=\left\{a_{1}, a_{2}, a_{4}, x_{2}\right\}$, with $\left\{x_{1}, x_{2}\right\}=\left\{a_{5}, a_{6}\right\}$. If $x_{1}=5$, then $f$ corresponds to $f_{3}$; if $x_{1}=6$, then $f$ is equivalent to $f_{3}$ by the involution $\alpha=\left(a_{5} a_{6}\right)$. The assertion follows. 


\subsection{Palette index and connected components}

Let $G_{1}, G_{2}, \ldots, G_{t}$ be the connected components of a graph $G$. It is obvious that

$$
\check{s}(G) \geq \max \left\{\check{s}\left(G_{i}\right): 1 \leq i \leq t\right\},
$$

that is, the palette index of $G$ is at least as bad as the palette index of the worst component. It is also clear that $\check{s}(G)$ could be strictly larger than the maximum among the palette indices of its components. Take for instance a graph $G$ given by the disjoint union of $t i$-regular class 1 graphs $G_{i}: \check{s}(G)=m$ whereas $\check{s}\left(G_{i}\right)=1$, for every $i=1, \ldots, m$. Looking at this example one could wonder whether $\check{s}(G)$ is effectively equal to the maximum among the palette indices $\check{s}\left(G_{i}\right)$, when the components $G_{i}$ are all $d$-regular and so $G$ itself is $d$ regular. It is not hard to verify that this is the case for 2 and 3-regular graphs. In this section we exhibit examples which prove that the situation is completely different for 4-regular graphs. One of the main reason is that the set of palettes used in an edge-coloring of a 4-regular graph with prescribed $\check{s}(G)$ is not uniquely determined (as happens for 2-regular and 3-regular graphs).

Proposition 3. Let $G$ be a 4-regular graph and let $G_{1}, G_{2}$ be connected components of $G$ such that $\check{s}\left(G_{1}\right)=\check{s}\left(G_{2}\right)=3 ; G_{1}$ has no perfect matching; $G_{2}$ has no even cycle decomposition. Then $4 \leq \check{s}(G) \leq 5$.

Proof. The graph $G$ is class 2 , since $G_{1}$ has no perfect matching; hence $\check{s}(G) \geq 3$. We show that $\check{s}(G)>3$. Suppose that $\check{s}(G)=3$, then $G$ has an even 2 -factor or an even cycle decomposition of size 3 , since Corollary 1 holds. That yields a contradiction, since $G_{1}$ has no even 2 -factor and $G_{2}$ has no even cycle decomposition. Hence, $4 \leq \check{s}(G) \leq 5$.

In Example 1 and 2 we use Proposition 3 to construct non-connected 4regular graphs with palette index 4 and 5 , respectively.

Example 1. The graph in Figure 2(a), say $G_{1}$, is a 4-regular graph with no perfect matching (its order is odd) and palette index 3 , since the graph $G_{1}$ admits the coloring $f_{2}$ defined in Proposition 1. The graph in Figure 3 is a 4-regular graph, say $G_{2}$, of class 2 since no perfect matching contains the edge $\left[u_{1}, v\right]$. The graph $G_{2}$ admits the coloring $f_{1}$ defined in Proposition 1 . The graph $G_{2}$ has no even cycle decomposition since it has a cut-vertex. By Proposition 3 , the palette index of the graph union $G=G_{1} \cup G_{2}$ is at least 4 . In Figure 4 it is shown that the graph $G$ admits the coloring $f_{3}$ defined in Proposition 2, that is, $\check{s}(G)=4$.

In the construction of a non-connected 4-regular graph with palette index 5 we use the graph in Figure 2(b). This graph is obtained by connecting three copies of the graph $H$ in Figure 6(a). The following statement holds for 4regular graphs with palette index 4 containing a subgraph isomorphic to $H$; it will be used in Example 2 and in the proof of Proposition 9. 


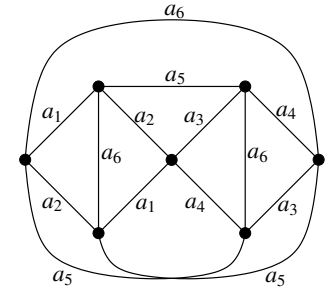

(a)

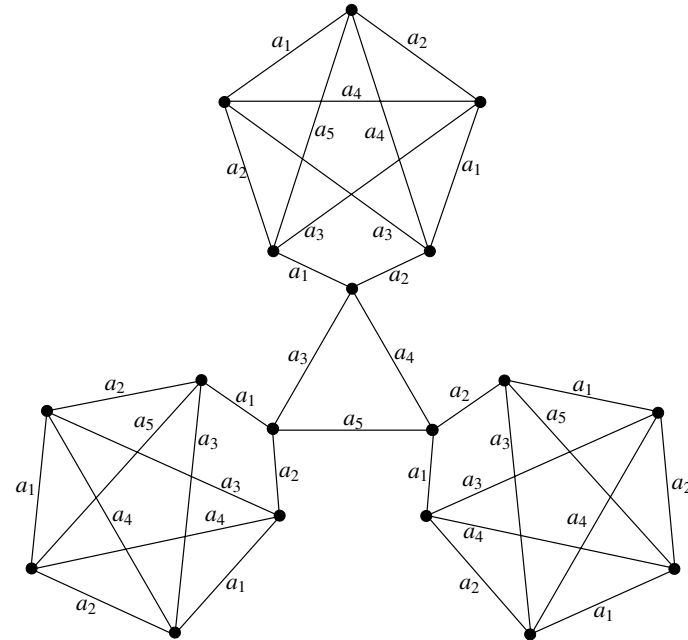

(b)

Figure 2: (a) A 4-regular with no perfect matching and palette index 3; (b) a 4-regular graph with no even cycle decomposition and palette index 3 .

Lemma 2. Let $H$ be the graph obtained by subdividing an edge of the complete graph $K_{5}$ with the insertion of a new vertex $u$. Let $G$ be a 4-regular graph with palette index 4 containing a subgraph isomorphic to $H$. If $G$ admits the $\check{s}$-minimal coloring $f_{3}$ with color-set $\mathcal{C}_{3}=\left\{a_{j}: 1 \leq j \leq 6\right\}$ and palettes $P_{1}=$ $\left\{a_{1}, a_{2}, a_{3}, a_{4}\right\}, P_{2}=\left\{a_{1}, a_{2}, a_{3}, a_{5}\right\}, P_{3}=\left\{a_{1}, a_{2}, a_{4}, a_{6}\right\}, P_{4}=\left\{a_{3}, a_{4}, a_{5}, a_{6}\right\}$, then $f_{3}$ induces a proper edge-coloring $\hat{f}$ in $H$ such that the vertex $u$ has palette $P_{\hat{f}}(u)=\left\{a_{3}, a_{6}\right\}$ or $P_{\hat{f}}(u)=\left\{a_{4}, a_{5}\right\}$ with respect to $\hat{f}$. Consequently, the vertex $u$ has palette $P_{f_{3}}(u)=\left\{a_{3}, a_{4}, a_{5}, a_{6}\right\}$ with respect to $f_{3}$.

Proof. The coloring $f_{3}$ induces a proper edge-coloring $\hat{f}$ in $H$; therefore, the palettes of the vertices in $V(H) \backslash\{u\}$ (with respect to $\hat{f}$ ) form a subset $\mathcal{P}_{\hat{f}}$ of $\mathcal{P}_{f_{3}}=\left\{P_{1}, P_{2}, P_{3}, P_{4}\right\}$. Since $H$ is class 2 , the set $\mathcal{P}_{\hat{f}}$ contains at least two elements; hence, there exists at least one palette of $\mathcal{P}_{\hat{f}}$ containing the 2 -subset $\left\{a_{1}, a_{2}\right\}$. The edges with the colors $a_{1}$ and $a_{2}$ form an even circuit $C$ in $H$. Since $|V(H)|=6$, the circuit $C$ has length 6 or 4 .

We show that $C$ has length 4 . Suppose that $C$ has length 6 , then the complementary subgraph of $C$ in $H$ is a circuit $C^{\prime}$ of length 5 . We denote by $A^{\prime}$ the set of distinct colors that $\hat{f}$ assigns to the edges of $C^{\prime}$. Since $C^{\prime}$ is class 2, the set $A^{\prime}$ contains at least three colors. Since the edges of $C$ are colored with $a_{1}$ and $a_{2}$, the set $A^{\prime}$ cannot contain the colors $a_{1}, a_{2}$, that is, $A^{\prime} \subseteq\left\{a_{3}, a_{4}, a_{5}, a_{6}\right\}$. If $A^{\prime}=\{a, b, c\} \subset\left\{a_{3}, a_{4}, a_{5}, a_{6}\right\}$, then $\hat{f}$ assigns one of the colors in $A^{\prime}$, say $c$, to exactly one edge of $C^{\prime}$ and assigns the color $a$ (respectively, $b$ ) to two non-adjacent edges of $C^{\prime}$. Consequently, the set $\mathcal{P}_{\hat{f}}$ contains 


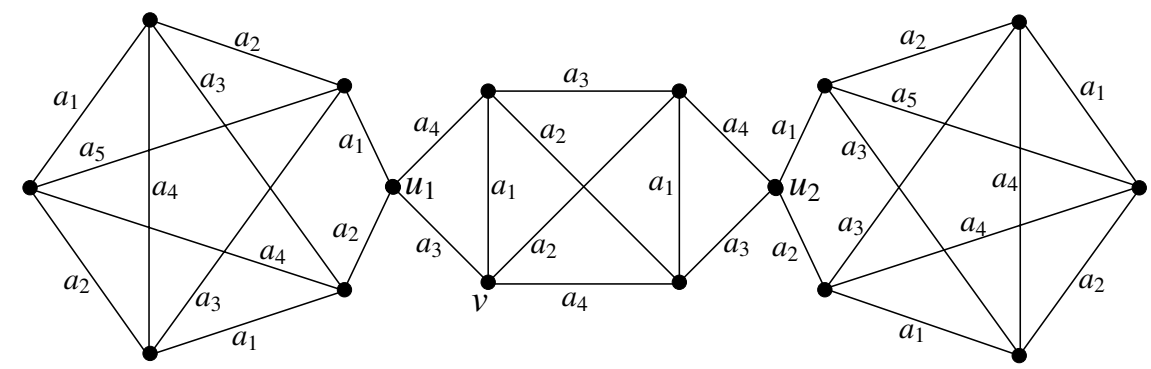

Figure 3: A 4-regular graph with no even cycle decomposition and palette index 3.
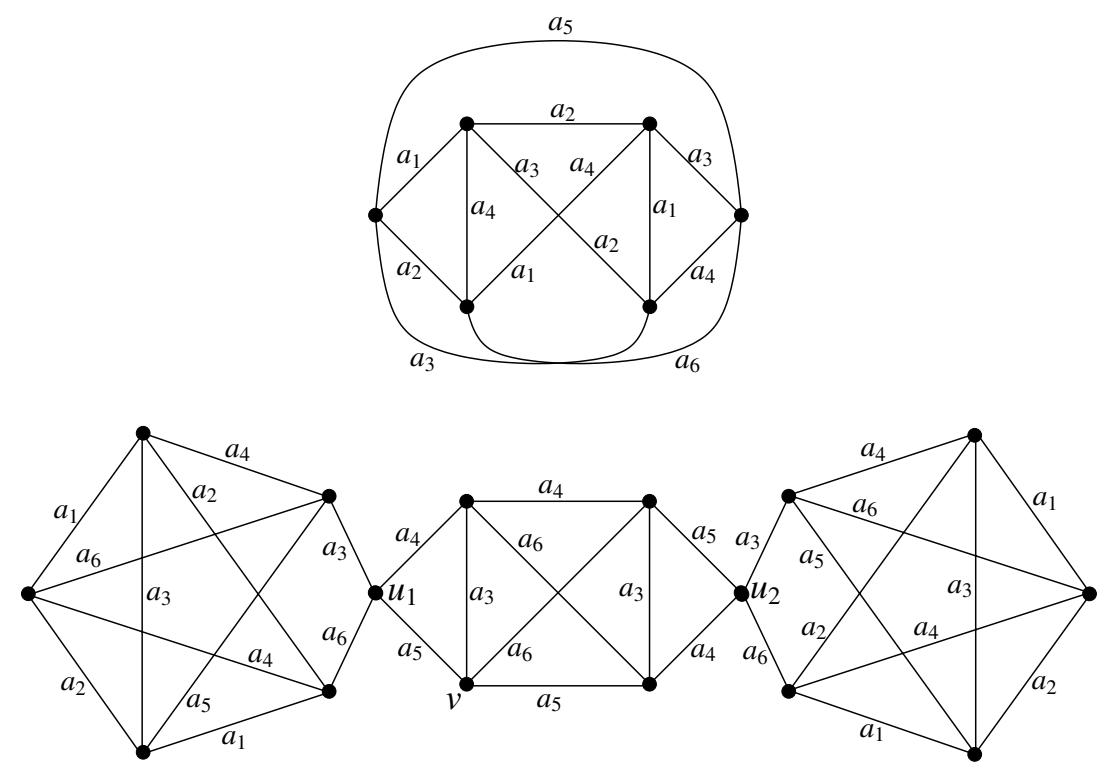

Figure 4: A non-connected 4-regular graph with palette index 4 and two connected components having palette index 3 . 
the palettes $\left\{a_{1}, a_{2}, a, b\right\},\left\{a_{1}, a_{2}, a, c\right\},\left\{a_{1}, a_{2}, b, c\right\}$. That yields a contradiction, since $\mathcal{P}_{\hat{f}} \subseteq \mathcal{P}_{f}$ and at least one of the palettes $\left\{a_{1}, a_{2}, a, b\right\},\left\{a_{1}, a_{2}, a, c\right\}$, $\left\{a_{1}, a_{2}, b, c\right\}$ does not belong to $\mathcal{P}_{f_{3}}$, for every $\{a, b, c\} \subset\left\{a_{3}, a_{4}, a_{5}, a_{6}\right\}$. Hence, $A^{\prime}=\left\{a_{3}, a_{4}, a_{5}, a_{6}\right\}$. Since $A^{\prime}$ has cardinality 4 and $C^{\prime}$ has length 5 , the coloring $\hat{f}$ assigns one of the colors in $A^{\prime}$, say $a$, to exactly two non-adjacent edges of $C^{\prime}$ and assigns each color $b \in A^{\prime}, b \neq a$, to exactly one edge of $C^{\prime}$. Consequently, the set $\mathcal{P}_{\hat{f}}$ contains at least one of the palettes $\left\{a_{1}, a_{2}, a_{3}, a_{6}\right\},\left\{a_{1}, a_{2}, a_{4}, a_{5}\right\}$. That yields a contradiction, since $\mathcal{P}_{\hat{f}} \subseteq \mathcal{P}_{f_{3}}$ and $\left\{a_{1}, a_{2}, a_{3}, a_{6}\right\},\left\{a_{1}, a_{2}, a_{4}, a_{5}\right\} \notin \mathcal{P}_{f_{3}}$. It is thus proved that $C$ cannot have length 6 , that is, $C$ has length 4 .

A circuit of length 4 in $H$ can pass through the vertex $u$ or not. We show that $u \notin V(C)$. Suppose that $u \in V(C)$. Without loss of generality, we can set $C=\left(u, v_{1}, v_{3}, v_{5}, u\right)$. Since the edges of $C$ are colored with $a_{1}$, $a_{2}$ and $C$ does not pass through the vertices $v_{2}, v_{4}$, the palette of $v_{2}$ and $v_{4}$ (with respect to $\hat{f}$ ) is $\left\{a_{3}, a_{4}, a_{5}, a_{6}\right\}$. We denote by $d \in\left\{a_{3}, a_{4}, a_{5}, a_{6}\right\}$ the color of the edge $\left[v_{2}, v_{4}\right]$ and set $\{a, b, c\}=\left\{a_{3}, a_{4}, a_{5}, a_{6}\right\} \backslash\{d\}$. Each color $\ell \in\{a, b, c\}$ induces a perfect matching $M_{\ell}$ in $H$ consisting of exactly two edges belonging to the set $E^{\prime}=\left\{\left[v_{2}, v_{i}\right],\left[v_{4}, v_{i}\right]: i=1,3,5\right\}$. The matchings $M_{a}, M_{b}, M_{c}$ partition the edges in $E^{\prime}$. This fact implies that the vertices $v_{1}, v_{3}, v_{5}$ have three distinct palettes, namely, the palettes $\left\{a_{1}, a_{2}, a, b\right\}$, $\left\{a_{1}, a_{2}, a, c\right\},\left\{a_{1}, a_{2}, b, c\right\}$. That yields a contradiction, since at least one of the palettes $\left\{a_{1}, a_{2}, a, b\right\},\left\{a_{1}, a_{2}, a, c\right\},\left\{a_{1}, a_{2}, b, c\right\}$ does not belong to $\mathcal{P}_{f_{3}}$, for every $\{a, b, c\} \subset\left\{a_{3}, a_{4}, a_{5}, a_{6}\right\}$. It is thus proved that $u \notin V(C)$.

Without loss of generality, we can set $C=\left(v_{2}, v_{3}, v_{4}, v_{5}\right)$. Since the edges of $C$ are colored with $a_{1}$ and $a_{2}$, the palettes of the vertices $v_{2}$ and $v_{4}$ share exactly three colors, namely, $P_{\hat{f}}\left(v_{2}\right) \cap P_{\hat{f}}\left(v_{4}\right)=\left\{a_{1}, a_{2}, a\right\}$ with $a \in\left\{a_{3}, a_{4}\right\}$. Therefore, $\left\{P_{\hat{f}}\left(v_{2}\right), P_{\hat{f}}\left(v_{4}\right)\right\}=\left\{P_{1}, P_{2}\right\}$ or $\left\{P_{\hat{f}}\left(v_{2}\right), P_{\hat{f}}\left(v_{4}\right)\right\}=\left\{P_{1}, P_{3}\right\}$. In the former case, the coloring $\hat{f}$ assigns the colors $a_{3}$ and $a_{6}$ to the edges $\left[v_{1}, v_{3}\right],\left[v_{1}, u\right]$, since $P_{\hat{f}}\left(v_{1}\right)=\left\{a_{3}, a_{4}, a_{5}, a_{6}\right\}$ and the edges $\left[v_{1}, v_{2}\right],\left[v_{1}, v_{4}\right]$ are colored with $a_{4}$ and $a_{5}$; if $\left[v_{1}, v_{3}\right]$ is colored with $a_{6}$, then the edge $\left[v_{3}, v_{5}\right]$ is colored with $a_{4}$, since the unique palette of $f_{3}$ containing $\left\{a_{1}, a_{2}, a_{6}\right\}$ is $P_{3}$; consequently, the edge $\left[v_{5}, u\right]$ is colored with $a_{6}$, since $\left[v_{1}, u\right]$ is colored with $a_{3}$ and the unique palettes of $f$ containing $\left\{a_{1}, a_{2}, a_{4}\right\}$ are $P_{1}$ and $P_{2}$. Therefore, $P_{\hat{f}}(u)=\left\{a_{3}, a_{6}\right\}$. The same arguments can be repeated when $\left[v_{1}, v_{3}\right]$ is colored with $a_{3}$ and also when $\left\{P_{\hat{f}}\left(v_{2}\right), P_{\hat{f}}\left(v_{4}\right)\right\}=\left\{P_{1}, P_{3}\right\}$. In this last case, the palette of $u$ with respect to $\hat{f}$ is $P_{\hat{f}}(u)=\left\{a_{4}, a_{5}\right\}$. It is straightforward to see that the palette of $u$ with respect to $f_{3}$ is $P_{f_{3}(u)}=\left\{a_{3}, a_{4}, a_{5}, a_{6}\right\}$, since the unique palette of $f_{3}$ containing $P_{\hat{f}}(u)$ is $P_{4}=\left\{a_{3}, a_{4}, a_{5}, a_{6}\right\}$.

Now we are in position to construct a non-connected 4-regular graph with palette index 5 .

Example 2. As already remarked in Example 1, the graph $G_{1}$ in Figure 2(a) has palette index 3 and no perfect matching. The graph in Figure $2(\mathrm{~b})$, say $G_{2}$, has three subgraphs isomorphic to $H$, say $H_{i}$, with $1 \leq i \leq 3$. For every $H_{i}$, we denote by $u_{i}$ the unique vertex of degree 2 in $H_{i}$. The graph $G_{2}$ has no even cycle decomposition, since it has a cut-vertex. Furthermore, it has palette index 3 and 
admits the coloring $f_{1}$ defined in Proposition 1. By Proposition 3 , the palette index of the graph union $G=G_{1} \cup G_{2}$ is at least 4 . We show that $\check{s}(G)>4$. Suppose that $\check{s}(G)=4$. Since Proposition 2 holds, the graph $G$ admits the coloring $f_{3}$. By Lemma 2 , the coloring $f_{3}$ induces a proper edge-coloring $\hat{f}$ in each $H_{i}$ such that the vertices $u_{i}$ have palette $\left\{a_{3}, a_{6}\right\}$ or $\left\{a_{4}, a_{5}\right\}$ with respect to $\hat{f}$ and palette $P_{4}=\left\{a_{3}, a_{4}, a_{5}, a_{6}\right\}$ with respect to $f_{3}$. Suppose that $u_{1}$ and $u_{2}$ have distinct palettes with respect to $\hat{f}$, then the edge $\left[u_{1}, u_{2}\right]$ is colored with $a_{1}$ or $a_{2}$, as $f_{3}$ is proper. That yields a contradiction since $u_{1}, u_{2}$ have palette $P_{4}=\left\{a_{3}, a_{4}, a_{5}, a_{6}\right\}$ and $a_{1}, a_{2} \notin P_{4}$. Hence $u_{1}, u_{2}$ have the same palette with respect to $\hat{f}$, say $\left\{a_{3}, a_{6}\right\}$. Since $u_{1}, u_{2}$ have palette $P_{4}=\left\{a_{3}, a_{4}, a_{5}, a_{6}\right\}$ with respect to $f_{3}$, the edges $\left[u_{1}, u_{2}\right],\left[u_{1}, u_{3}\right],\left[u_{2}, u_{3}\right]$ are colored with the colors $a_{4}$, $a_{5}$. That yields a contradiction, since $f$ is proper.

In the following statements we highlight some connections between the (edge) connectivity of the graph and its palette index.

Proposition 4. Let $G$ be a 4-regular graph with a perfect matching, then $\check{s}(G)=$ 1,3 or 4 .

Proof. Denote by $M$ a perfect matching of $G$ and by $G^{\prime}$ the complementary cubic subgraph of $M$ in $G$. By [7, Theorem 9], $\check{s}\left(G^{\prime}\right) \in\{1,3,4\}$. Denote by $f^{\prime}$ an edge-coloring of $G^{\prime}$ with $\check{s}\left(G^{\prime}\right)$ palettes. We color all the edges of $M$ with the same color $a$ (not belonging to the palettes of $f^{\prime}$ ) and add $a$ to the palettes of $f^{\prime}$. We obtain an edge-coloring of $G$ with $\check{s}\left(G^{\prime}\right)$ palettes, whence $\check{s}(G) \leq \check{s}\left(G^{\prime}\right)$.

Corollary 2. Let $G$ be a 4-regular graph with palette index 5. Then, either $G$ has a connected component of odd order or all connected components of $G$ are even and $G$ is not 4-edge-connected.

Proof. The graph $G$ has no perfect matching, otherwise by Proposition 4 $\check{s}(G) \leq 4$. Suppose that all components of $G$ are even and 4-edge-connected, then $G$ has a perfect matching, since [12, Theorem 2.2] holds. The at least one component either is odd or is not 4-edge-connected.

Proposition 5. Let $G$ be a 4-regular graph having a connected component with no perfect matching and a cut-vertex. Then $4 \leq \check{s}(G) \leq 5$.

Proof. The graph $G$ is not a class 1 graph because it has no perfect matching, hence $\check{s}(G) \geq 3$. Furthermore, $G$ has not an even cycle decomposition since it has a cut-vertex and $G$ has no even 2-factor ( $G$ has no perfect matching). It follows by Corollary 1 that $\check{s}(G)>3$.

\subsection{Connected 4-regular graphs with palette index 4 and 5.}

As we have already remarked, the existence of 4-regular graphs with palette index 1 and 3 can be easily proved and graphs with palette index 3 can be used 
to construct non-connected 4-regular graphs with palette index 4 and 5 (see Example 1 and 2).

In this section we consider the connected case. As remarked in Section 1, connected 4-regular graphs with palette index $>3$ seem to be rare. We distinguish two types of 4-regular graphs with palette index 4: those who have and those who have not a perfect matching.

In Proposition 6 we shall see that the existence of 4-regular graphs with no perfect matching and palette index 4 can be easily obtained. The existence of 4-regular graphs with a perfect matching and palette index 4 or of 4-regular graphs with palette index 5 is harder to prove. We give some examples in Proposition 8 and 9. We also note that a 4-regular graph with palette index $>3$ has no even 2-factor, nevertheless the non-existence of even 2 -factors does not imply that the palette index is bigger than 3 (see Proposition 7 ).

The complete graph $K_{5}$ is the smallest example of 4-regular graph with no perfect matching and palette index 4 (the palette index of $K_{5}$ is calculated in [7]). In the following proposition we show that $K_{5}$ can be used to construct other examples of 4-regular graphs with no perfect matching and palette index 4.

Proposition 6. Let $K_{5}$ be the complete graph on the vertices $\left\{u_{i}: 1 \leq i \leq 5\right\}$ and let $G$ be a class 1 4-regular graph. Delete an edge in $K_{5}$, say $e=\left[u_{1}, u_{2}\right]$, and an edge in $G$, say $e^{\prime}=\left[w_{1}, w_{2}\right]$; connect $K_{5}-e$ and $G-e^{\prime}$ by adding the edges $\left[u_{1}, w_{1}\right],\left[u_{2}, w_{2}\right]$. The resulting graph is a 4-regular graph with no perfect matching and palette index 4.

Proof. Denote by $G^{\prime}$ the 4-regular graph obtained by connecting $K_{5}$-e and $G-e^{\prime}$. The graph $G^{\prime}$ is class 2 because it has no perfect matching (its order is odd); hence $\check{s}\left(G^{\prime}\right) \geq 3$. We show that $\check{s}\left(G^{\prime}\right)>3$. Suppose that $\check{s}\left(G^{\prime}\right)=3$. Since $G^{\prime}$ has no perfect matching and Corollary 1 holds, the graph $G^{\prime}$ has an even cycle decomposition of size 3 . That yields a contradiction since a graph having $K_{5}-e$ as induced subgraph has no even cycle decomposition. Therefore $\check{s}\left(G^{\prime}\right)>3$.

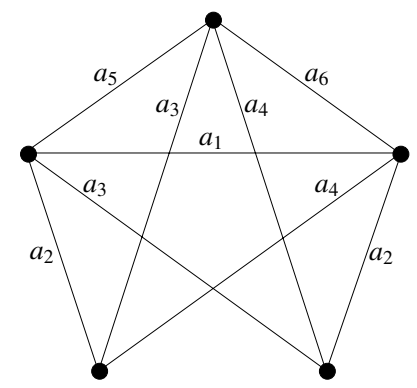

Figure 5: An edge-coloring of $K_{5}-e$.

We show that $\check{s}\left(G^{\prime}\right)=4$. The graph $G$ has palette index 1 , since $G$ is class 
1. Without loss of generality we can say that every vertex of $G$ has palette $P_{1}=\left\{a_{1}, a_{2}, a_{3}, a_{4}\right\}$ and the edge $e^{\prime}$ has color $a_{1}$. The edges of $K_{5}$-e can be colored as in Figure 5 . We color the edges $\left[u_{1}, w_{1}\right]$, $\left[u_{2}, w_{2}\right]$ with $a_{1}$. We obtain an edge-coloring of $G^{\prime}$ with exactly 4 palettes; therefore $\check{s}\left(G^{\prime}\right)=4$.

A 4-regular graph, with a perfect matching and palette index 4 , has no pair of edge-disjoint perfect matchings, that is, no even 2-factor. This fact also follows from the proof of Proposition 4. The non-existence of even 2-factors in a 4-regular graph $G$ with a perfect matching does not guarantee that $G$ has palette index 4 . It might well happen that $G$ has palette index 3 and admits the coloring $f_{2}$ defined in Proposition 1. This is the case of the graph $\bar{G}$ in Figure 7 .

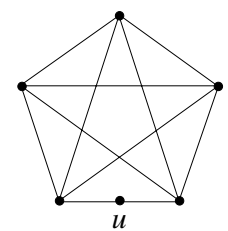

(a)

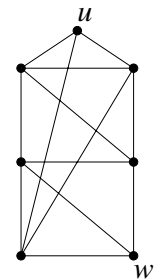

(b)

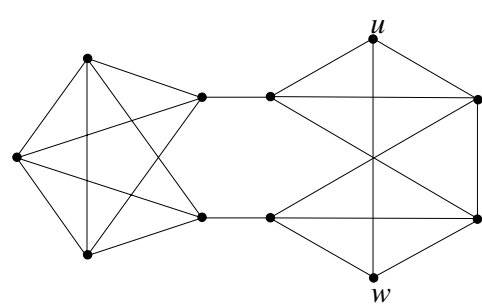

(c)

Figure 6: (a) The graph $H$. (b) The graph $J$. (c) The graph $K$.

The graph $\bar{G}$ can be constructed as follows: consider four copies of the graph $J$ in Figure 6(b), say $J_{i}$ with $1 \leq i \leq 4$; label the vertices $u$, $w$ of each copy $J_{i}$ by $u_{i}, w_{i}$, respectively; connect the graphs $J_{i}$ and two new vertices $u_{0}, w_{0}$ by adding the edges $\left[w_{1}, w_{2}\right],\left[w_{3}, u_{0}\right],\left[w_{0}, w_{4}\right],\left[u_{0}, w_{0}\right],\left[u_{0}, u_{1}\right],\left[u_{0}, u_{4}\right],\left[w_{0}, u_{2}\right]$, $\left[w_{0}, u_{3}\right]$. The resulting graph $\bar{G}$ is 4-regular of order 30 . The following statement holds.

Proposition 7. The graph $\bar{G}$ has a perfect matching and no even 2-factor. Furthermore, the graph $\bar{G}$ admits the $\check{s}$-minimal coloring $f_{3}$ with color-set $\mathcal{C}_{3}=$ $\left\{a_{j}: 1 \leq j \leq 6\right\}$ and palettes $P_{1}=\left\{a_{1}, a_{2}, a_{3}, a_{4}\right\}, P_{2}=\left\{a_{1}, a_{2}, a_{5}, a_{6}\right\}$, $P_{3}=\left\{a_{3}, a_{4}, a_{5}, a_{6}\right\}$, that is, $\bar{G}$ has palette index 3 .

Proof. A perfect matching in $\bar{G}$ can be constructed by taking a perfect matching in each graph $J_{i}-\left\{w_{i}\right\}$ together with the edges $\left[w_{1}, w_{2}\right]$, [ $\left.u_{0}, w_{3}\right]$, $\left[w_{0}, w_{4}\right]$. We show that $\bar{G}$ has no even 2 -factor, that is, $\bar{G}$ has no pair of edgedisjoint perfect matchings. To this end we prove that every perfect matching of $\bar{G}$ contains the edge $\left[w_{1}, w_{2}\right]$. Suppose that $M$ is a perfect matching of $\bar{G}$ not containing the edge $\left[w_{1}, w_{2}\right]$. Since $\left[w_{1}, w_{2}\right] \notin M$ and $J_{1}, J_{2}$ have odd order, the matching $M$ contains the edges $\left[u_{0}, u_{1}\right],\left[w_{0}, u_{2}\right]$ and consequently it also contains a perfect matching of $J_{3}$. That yields a contradiction, since $J_{3}$ has odd order. It is thus proved that every perfect matching of $\bar{G}$ contains the edge $\left[w_{1}, w_{2}\right]$, that is, $\bar{G}$ has no pair of edge-disjoint perfect matchings. 


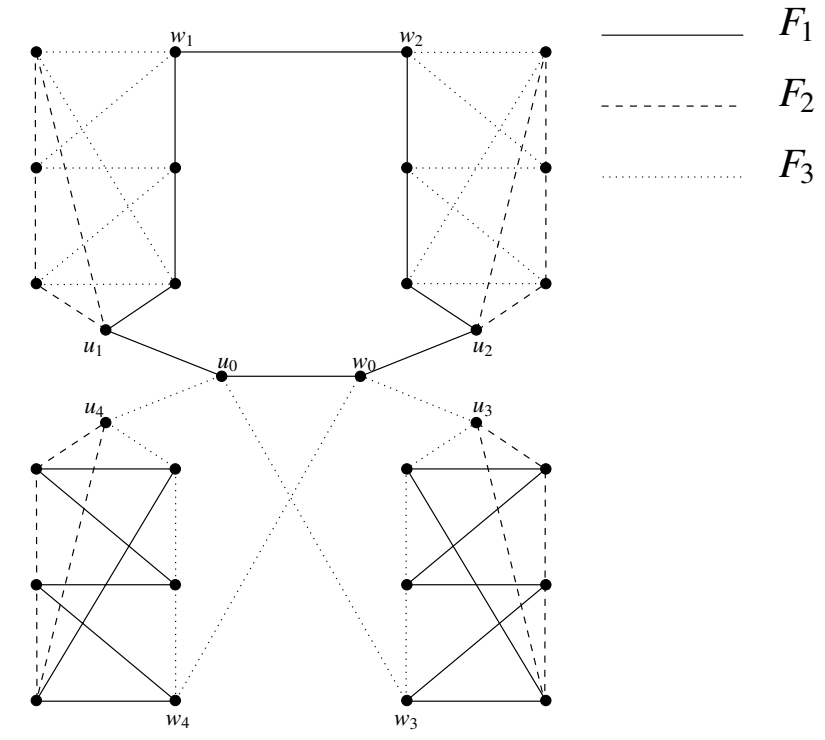

Figure 7: An even cycle decomposition of $\bar{G}$ of size 3 .

We show that $\bar{G}$ admits the coloring $f_{2}$ defined in Proposition 1. This is equivalent to show that the edge-set of $\bar{G}$ can be partitioned into three even cycles, say $F_{1}, F_{2}, F_{3}$ (the edges of each cycle $F_{i}$, with $1 \leq i \leq 3$, can be colored alternately with the colors $a_{2 i-1}$ and $a_{2 i}$ ). The subgraphs $F_{1}, F_{2}, F_{3}$ can be defined as in Figure 7. The assertion follows.

We slightly change the construction of $\bar{G}$ and obtain a 4-regular graph with a perfect matching and palette index 4 . More specifically, we replace the subgraph $J_{4}$ of $\bar{G}$ with the graph $K$ in Figure 6(c) and denote by $\tilde{G}$ the 4-regular graph thus obtained (see Figure 8).

Proposition 8. The graph $\tilde{G}$ has a perfect matching and palette index 4 .

Proof. As for the graph $\bar{G}$, every perfect matching of $\tilde{G}$ contains the edge $\left[w_{1}, w_{2}\right]$; therefore $\tilde{G}$ has no even 2 -factor. The non-existence of an even 2-factor implies that $\tilde{G}$ is class 2 and no $\breve{s}$-minimal coloring of $\tilde{G}$ is equivalent to the coloring $f_{1}$ defined in Proposition 1. Therefore, $\check{s}(\tilde{G}) \geq 3$ and if $\check{s}(\tilde{G})=3$, then $\tilde{G}$ has an even cycle decomposition of size 3 (see Corollary 1 ). The graph $\tilde{G}$ has no even cycle decomposition because the graph $K$ contains a subgraph isomorphic to $K_{5}-e$ (the complete graph $K_{5}$ with an edge deleted). Therefore $\check{s}(\tilde{G}) \geq 4$. Since $\tilde{G}$ has a perfect matching (see Figure 8) and Proposition 4 holds, the palette index is 4 .

We construct connected 4-regular graphs with palette index 5 . Consider two copies of the graph $H$ in Figure 6(a), say $H_{1}$ and $H_{2}$. For $i=1,2$, denote by $u_{i}$ the unique vertex of degree 2 in $H_{i}$. Connect the graphs $H_{1}$ and $H_{2}$ to a new 


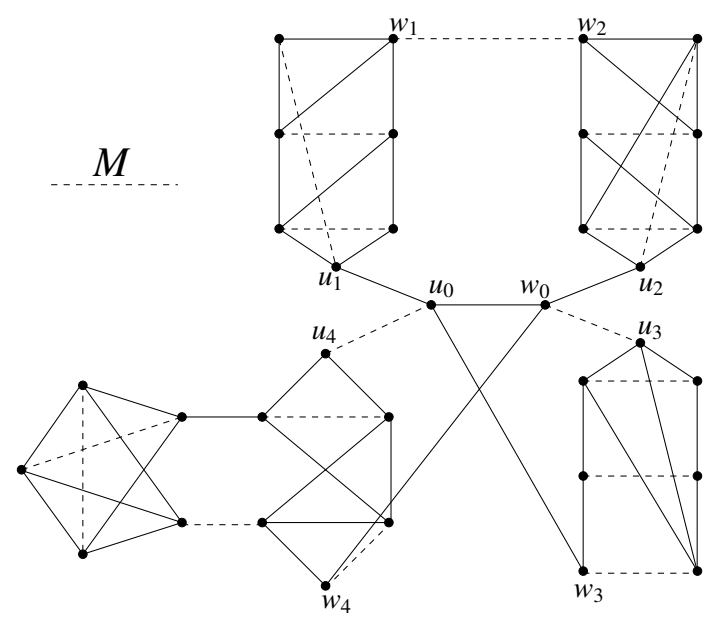

Figure 8: The graph $\tilde{G}$ (dashed edges form a perfect matching $M$ of $\tilde{G}$ ).

vertex $w$ by adding the edges $\left[u_{i}, w\right]$, with $i=1,2$. Also add the edge $\left[u_{1}, u_{2}\right]$ and denote by $L^{*}$ the graph thus obtained. The following statement holds.

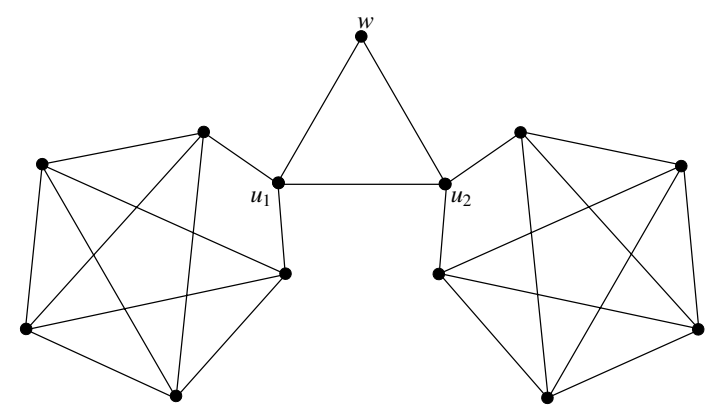

Figure 9: The graph $L^{*}$ used in the proof of Proposition 9.

Proposition 9. Let $G_{1}$ and $G_{2}$ be graphs of even order with all vertices of degree 4 but one of degree 2 , say $w_{1}$ and $w_{2}$, respectively. Let $G^{*}$ be the graph obtained from the graph union $G^{*}=G_{1} \cup G_{2} \cup L^{*}$ by adding the edges [w, $\left.w_{1}\right]$, $\left[w, w_{2}\right],\left[w_{1}, w_{2}\right]$. The graph $G^{*}$ has palette index 5 .

Proof. The graph $G^{*}$ is class 2 , as its order is odd. Therefore, $\check{s}(G) \geq$ 3. Since the graph $G^{*}$ has no even circuit passing through the vertex $w$ and Corollary 1 holds, the palette index of $G^{*}$ is at least 4 .

Suppose that $\check{s}(G)=4$, then we can color the edges of $G^{*}$ using the coloring $f_{3}$ defined in Proposition 1. By Lemma 2, the vertices $u_{1}$ and $u_{2}$ have 


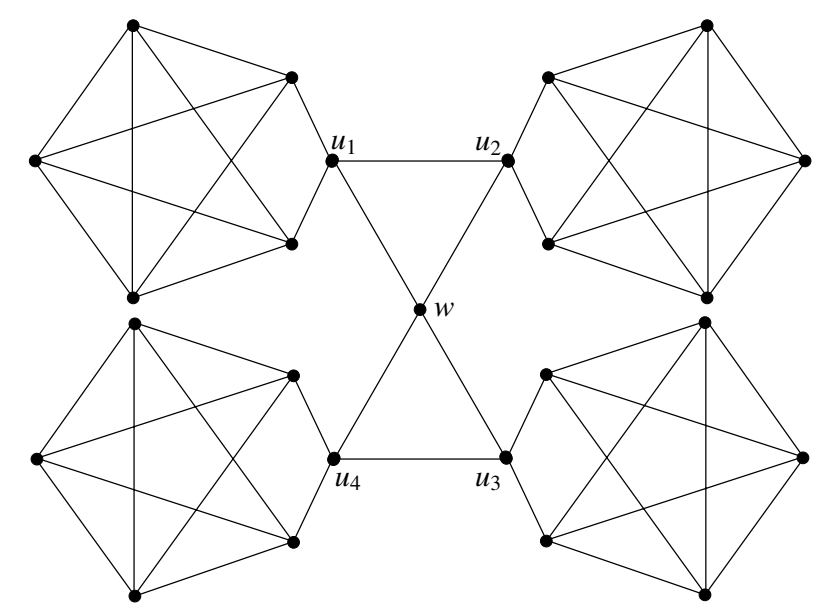

Figure 10: A connected 4-regular graph with palette index 5.

palette $P_{4}=\left\{a_{3}, a_{4}, a_{5}, a_{6}\right\}$ with respect to $f_{3}$. By the same lemma, the coloring $f_{3}$ induces a coloring $\hat{f}$ in $H_{1}$ and $H_{2}$ such that $\left\{P_{\hat{f}}\left(u_{1}\right), P_{\hat{f}}\left(u_{2}\right)\right\} \subseteq$ $\left\{\left\{a_{3}, a_{6}\right\},\left\{a_{4}, a_{5}\right\}\right\}$. If $P_{\hat{f}}\left(u_{1}\right) \neq P_{\hat{f}}\left(u_{2}\right)$, then we cannot color the edge $\left[u_{1}, u_{2}\right]$ using the colors in $P_{4}=\left\{a_{3}, a_{4}, a_{5}, a_{6}\right\}$. Therefore $P_{\hat{f}}\left(u_{1}\right)=P_{\hat{f}}\left(u_{2}\right)$. Without loss of generality, we can set $P_{\hat{f}}\left(u_{1}\right)=P_{\hat{f}}\left(u_{2}\right)=\left\{a_{3}, a_{6}\right\}$, whence $f_{3}\left(\left[u_{1}, u_{2}\right]\right)$, $f_{3}\left(\left[u_{1}, w\right]\right), f_{3}\left(\left[u_{2}, w\right]\right) \in\left\{a_{4}, a_{5}\right\}$, that is, the coloring $f_{3}$ assigns the same color to at least two adjacent edges. That yields a contradiction, since $f_{3}$ is proper. It it thus proved that $\check{s}\left(G^{*}\right)=5$.

In Figure 10 we show a connected 4-regular graph with palette index 5 that can be obtained from Proposition 9 by taking the graphs $G_{1}$ and $G_{2}$ equal to the graph $H$ in Figure 6(a).

\section{Palette index and even cycle decompositions.}

A regular graph of even degree $2 r$ and palette index 1 (class 1) always possesses an even cycle decomposition of size $r$ (each cycle is an even 2-factor obtained as the union of two different color classes of a minimum edge-coloring). We consider class 2 regular graphs. By Corollary 1, a 4-regular graph with no perfect matching and palette index 3 has an even cycle decomposition of size 3 . In Proposition 10 we extend this property to a family of $4 r$-regular graphs.

Proposition 10. Let $G$ be a 4 -regular graph, $r \geq 1$, with no perfect matching and palette index 3 . Then $G$ possesses an even cycle decomposition of size $3 r$.

Proof. Let $f$ be an edge-coloring of $G$ having three palettes, say $P_{1}, P_{2}$ and $P_{3}$. For $i=1,2,3$, denote by $V_{i}$ the set of vertices of $G$ having palette $P_{i}$. Given a palette $P_{i}$, we show that every color $a \in P_{i}$ is contained in exactly 
one of the other two palettes of $f$ distinct from $P_{i}$. We first show that the set $P_{i} \backslash\left(P_{j} \cup P_{k}\right)$, with $\{i, j, k\}=\{1,2,3\}$, is empty.

Suppose that there exists a color $a$ in $P_{i}$ which does not belong to $P_{j} \cup P_{k}$. The edges colored with $a$ form a perfect matching $M_{a}$ of the subgraph $G\left[V_{i}\right]$ $\left(G\left[V_{i}\right]\right.$ is the subgraph of $G$ induced by the vertices in $\left.V_{i}\right)$. If there exists a color $b$ in $P_{j} \cap P_{k}$, then the edges colored with $b$ form a perfect matching $M_{b}$ in the subgraph $G\left[V_{j} \cup V_{k}\right]$ and the set $M_{a} \cup M_{b}$ is a perfect matching in $G$, a contradiction. Therefore, $P_{j} \cap P_{k}=\emptyset$. Since $P_{j} \neq P_{i}$ and $P_{k} \neq P_{i}$, there exists at least one color $b \in P_{j} \backslash P_{i}$ and at least one color $c \in P_{k} \backslash P_{i}$. The color $b$ (respectively, $c$ ) does not belong to $P_{k}$ (respectively, to $P_{j}$ ) since $P_{j} \cap P_{k}=\emptyset$. Therefore the edges colored with $b$ (respectively, with $c$ ) form a perfect matching $M_{b}$ (respectively, $M_{c}$ ) in $G\left[V_{j}\right]$ (respectively, in $G\left[V_{k}\right]$ ). Then $M_{a} \cup M_{b} \cup M_{c}$ is a perfect matching in $G$, a contradiction. It is thus proved that $P_{i} \backslash\left(P_{j} \cup P_{k}\right)=\emptyset$, that is, every color $a \in P_{i}$ is contained in at least one of the other two palettes $P_{j}, P_{k}$. Since $G$ has no perfect matching, every color $a \in P_{i}$ is contained in exactly one of the two palettes $P_{j}, P_{k}$. We can thus write $P_{i}$ has the disjoint union $P_{i}=\left(P_{i} \cap P_{j}\right) \dot{\cup}\left(P_{i} \cap P_{k}\right)$ and set $\left|P_{i} \cap P_{j}\right|=h>0,\left|P_{i} \cap P_{k}\right|=4 r-h$, whence $4 r=\left|P_{k}\right|=2(4 r-h)$, that is, $h=2 r$. We have thus proved that every pair of palettes share $2 r$ colors, therefore we can write the palettes $P_{1}$, $P_{2}, P_{3}$ as follows: $P_{1}=\left\{a_{i}, b_{i} \mid 1 \leq i \leq 2 r\right\}, P_{2}=\left\{a_{i}, c_{i} \mid 1 \leq i \leq 2 r\right\}$ and $P_{3}=\left\{b_{i}, c_{i} \mid 1 \leq i \leq 2 r\right\}$.

We construct an even cycle decomposition of $G$. For every $i=1, \ldots, 2 r$, the edges colored with $a_{i}, b_{i}, c_{i}$ form a perfect matching $A_{i}, B_{i}, C_{i}$, respectively, in the subgraph $G\left[V_{1} \cup V_{2}\right], G\left[V_{1} \cup V_{3}\right], G\left[V_{2} \cup V_{3}\right]$, respectively. Then $A_{i} \cup A_{i+r}$, $B_{i} \cup B_{i+r}, C_{i} \cup C_{i+r}$, with $1 \leq i \leq r$, are even cycles of $G\left[V_{1} \cup V_{2}\right], G\left[V_{1} \cup V_{3}\right]$, $G\left[V_{2} \cup V_{3}\right]$, respectively. The set $\left\{A_{i} \cup A_{i+r}, B_{i} \cup B_{i+r}, C_{i} \cup C_{i+r} \mid 1 \leq i \leq r\right\}$ is an even cycle decomposition of $G$ of size $3 r$.

Proposition 10 can be inverted in the case of 4-regular graphs as follows.

Proposition 11. Let $G$ be a 4-regular graph with an even cycle decomposition of size 3 , then $\check{s}(G) \leq 3$.

Proof. Let $\mathcal{E}=\left\{F_{1}, F_{2}, F_{3}\right\}$ be an even cycle decomposition of $G$. Since the elements of $\mathcal{E}$ are even cycles, we can color alternately the edges of each $F_{i}$, $1 \leq i \leq 3$, with exactly two colors, say $a_{2 i-1}$ and $a_{2 i}$. In this way we define an edge-coloring $f: \mathcal{C} \rightarrow E(G)$ with color-set $\mathcal{C}=\left\{a_{j}: 1 \leq j \leq 6\right\}$. The coloring $f$ has palettes $P_{1}=\left\{a_{1}, a_{2}, a_{3}, a_{4}\right\}, P_{2}=\left\{a_{1}, a_{2}, a_{5}, a_{6}\right\}, P_{3}=\left\{a_{3}, a_{4}, a_{5}, a_{6}\right\}$, since a vertex of $G$ belongs to exactly two elements of $\mathcal{E}$. Therefore $G$ has palette index $\leq 3$.

Not all the graphs with palette index 3 have an even cycle decomposition. See for instance the graph in Figure 3 and the discussion in Example 1.

The graph in Figure 3 and Proposition 11 show that the family of 4-regular graphs with an even cycle decomposition of size $\leq 3$ is strictly contained in the class of 4-regular graphs with palette index $\leq 3$. We do not know whether the whole family of 4-regular graphs with an even cycle decomposition is strictly contained in the family of 4 -regular graphs with palette index $\leq 3$. The construction of a counterexample, that is, the construction of a 4-regular graph with 
an even cycle decomposition and palette index $>3$, seems to be hard: if this graph exists, then it has no even cycle decomposition of size less than 3 , since Proposition 11 holds; by the result in [9] and the conjecture in [10], 4-regular graphs with even cycle decompositions of size $>3$ do not seem easy to find (see the remarks in Section 1).

\section{Open problems.}

As remarked in Section 1, the palette index of a $d$-regular graph $G$ of class 2 satisfies the inequalities $3 \leq \check{s}(G) \leq d+1$. For $2 \leq d \leq 4$ we know that there exists a (connected) $d$-regular graph with palette index $d+1$ : it suffices to consider a cycle with at least one circuit of odd length for $d=2$; a cubic graph with no perfect matching for $d=3$ (see [7]); the graphs constructed in Proposition 9 for $d=4$. We leave as an open problem the construction of a (connected) $d$-regular graph with palette index $d+1$ for every integer $d \geq 5$.

Differently from the regular case, the palette index of a non-regular graph $G$ satisfies the inequalities $2 \leq \check{s}(G) \leq 2^{\Delta(G)+1}$ : since $G$ is non-regular, it has at least two vertices with different degree, that is, every proper edge-coloring of $G$ has at least two palettes of different cardinality; hence $\check{s}(G) \geq 2$; by Vizing's Theorem, we can find a proper edge-coloring $f$ of $G$ whose color-set $\mathcal{C}$ contains $\chi^{\prime}(G) \leq \Delta(G)+1$ colors, that is, the set $\mathcal{P}_{f}$ is a subset of the power-set of $\mathcal{C}$; hence $\check{s}(G) \leq\left|\mathcal{P}_{f}\right| \leq 2^{\Delta(G)+1}$. In a forthcoming paper the authors study the relationship between the palette index and the maximum degree $\Delta(G)$. They also consider the problem of constructing a non-regular graph with palette index $2^{\Delta(G)+1}($ see $[3])$.

In Sections 2.2 and 2.3 we have constructed 4-regular graphs with palette index 4 and 5 . None of our examples has an even cycle decomposition. It would be interesting to prove the existence (or non-existence) of a 4-regular graph with palette index $>3$ and an even cycle decomposition. This problem seems to be related to the problem of finding a 4-regular graph with all even cycle decompositions of size $>3$. As far as we know, this general problem about the size of an even cycle decomposition has never been considered before. To find results in this direction, we are investigating some families of 4-regular graphs, in particular, the family of line graphs of cubic graphs, [2].

\section{References}

[1] J.A. Bondy, U.S.R. Murty, Graph Theory, Springer, 2008.

[2] S. Bonvicini, From the palette index to the existence of even 2-factors and even cycle decompositions in the line graph of a cubic graph, preprint.

[3] S. Bonvicini, G. Mazzuoccolo, Palette index of non-regular graphs, preprint.

[4] A.C. Burris, R.H. Schelp, Vertex-distinguishing proper edge-colourings, J. Graph Theory 26 (1997), 73-82. 
[5] P. Erdös, Z. Füredi, A. Hajnal, P. Komjáth, V. Rödl, Á. Seress, Coloring graphs with locally few colors, Discrete Math. 59 (1986), 21-34.

[6] K. Edwards, M. Horňák, M. Woźniak, On the neighbour-distinguishing index of a graph, Graphs Combin. 22 (2006), 341-350.

[7] M. Horňák, R. Kalinowski, M. Meszka, M. Woźniak, Edge colorings and the number of palettes, Graphs Combin. 30 (2014), 619-626.

[8] J. Korner, C. Pilotto, G. Simonyi, Local chromatic number and Sperner capacity, J. Combin. Theory Ser. B 95 (2005), 101-117.

[9] J.H. Kim, N.C. Wormald, Random matchings which induce Hamilton cycles and Hamiltonian decompositions of random regular graphs, J. Combin. Theory Ser. B 81 (2001), 20-44.

[10] K. Markström, Even cycle decompositions of 4-regular graphs and line graphs, Discrete Math. 312 (2012), 2676-2681.

[11] R. W. Robinson, N. C. Wormald, Almost all regular graphs are Hamiltonian, Random Structures Algorithms 5 (1994), 363-374.

[12] S. O, D. B. West, Matching and edge-connectivity in regular graphs, European J. Combin. 32 (2011), 324-329. 